\title{
Investigation of harmful slip in a rolling bearing using a thermographic camera with a derotator
}

Altmann, Bettina, Reithmeier, Eduard

Bettina Altmann, Eduard Reithmeier, "Investigation of harmful slip in a rolling bearing using a thermographic camera with a derotator," Proc. SPIE 11503, Infrared Sensors, Devices, and Applications X, 115030R (22 August 2020); doi: $10.1117 / 12.2568036$ 


\title{
Investigation of Harmful Slip in a Rolling Bearing Using a Thermographic Camera With a Derotator
}

\author{
Bettina Altmann ${ }^{\mathrm{a}}$ and Eduard Reithmeier ${ }^{\mathrm{a}}$ \\ ${ }^{a}$ Institute of Measurement and Automatic Control, Leibniz Universität Hannover, Nienburger \\ Str. 17, 30167 Hannover, Germany
}

\begin{abstract}
Due to their high availability and versatility, rolling bearings are a standard solution for mounting and support of rotating components. The service life of an entire rotating machine is often limited by the service life of rolling bearings. This can be shorter than expected if the rolling bearing is operated in harmful operating conditions, e.g. in the presence of slip. Slip means that there is a deviation between the theoretical angular velocity of the rolling element set and the actual angular velocity. In this context, slip is harmful if it leads to increased friction and thus heating of the bearing. The occurrence and properties of slip are not completely understood yet. Therefore, it is of interest to investigate a relationship between slip and heating of the rolling bearing in order to better understand damages of the rolling bearing. In this work, a method is presented in which, in addition to slip measurements with a high-speed camera, a thermal imaging camera is used to investigate the heating of the bearing during operation. Since the rotational movement and exposure time of the camera would cause motion blur, the thermal imaging camera is operated together with a derotator to optically eliminate the rotational movement. The investigations of the rolling bearing are carried out under different operating conditions (different loads and rotational speeds), which have an influence on the slip behavior of the bearing. Thus, the potential of this investigation method for deepening the understanding of heating and friction in rolling bearings is demonstrated.
\end{abstract}

Keywords: Roller bearing slip, thermographic inspection, optomechanical image derotator, contactless measurements, cylindrical roller bearings

\section{INTRODUCTION}

Rolling bearings are omnipresent in modern technology: over 100 billion rolling bearings are in use worldwide. ${ }^{1}$ The vast majority of bearings in rotating machines, vehicles, plants and tools are equipped with rolling bearings. The bearing performs two main tasks. ${ }^{2}$ The 'carrying' function focuses on the transmission of forces and moments between parts that move relative to each other. With the 'guiding' function, the aim is to determine the mutual position of the parts moving relative to each other as accurately as possible.

Optimal energy efficiency and high performance demand that the movement is as low-friction as possible. In rolling bearings, low friction is achieved by the rolling elements (some are held at a distance by cages). The rolling elements reduce frictional resistance since they replace pure sliding by rolling with a small sliding component.

In certain rotational speed and load ranges, the rolling component can recede and increased sliding can occur, so that the circumferential speed of the rolling partners varies. This is known as slip. ${ }^{1}$ In the circumferential direction deviations in the rotational speed of the rolling element set or cage are referred to as cage slip. Slip is not always harmful. ${ }^{3}$ Cage slip, however, is usually considered critical, since it can lead to failure long before the nominal life is reached. ${ }^{4}$ These failures can then in turn lead to machine failure which can lead to high costs or, in the worst case, to danger for people and the environment. ${ }^{1}$

The majority of bearing failures are generally due to inadequate lubrication, since an intact lubricating film in the rolling bearing is a basic requirement for low-wear operation of the rolling bearing. ${ }^{1}$ Slip as well as other potentially damaging rolling bearing behavior lead to the disruption of the ideal lubrication condition. ${ }^{3}$

Further author information:

E-mail: bettina.altmann@imr.uni-hannover.de, Telephone: +49511 7625817

Infrared Sensors, Devices, and Applications X, edited by Ashok K. Sood, Priyalal Wijewarnasuriya,

Arvind I. D'Souza, Proc. of SPIE Vol. 11503, 115030R - (c) 2020 SPIE

CCC code: $0277-786 \mathrm{X} / 20 / \$ 21 \cdot$ doi: $10.1117 / 12.2568036$

Proc. of SPIE Vol. $11503115030 \mathrm{R}-1$ 
An actually occurring lack of lubrication leads to increased friction, which in turn manifests itself in a rise in temperature. ${ }^{1}$ The temperature is therefore a good indicator for monitoring the lubrication condition.

Slip-induced damage is therefore best determined by measuring the extent of slip on the one hand, and by using the temperature of the bearing to draw conclusions about the extent of friction on the other hand. Measurement methods that measure slip or temperature are often contact-based and sometimes require modifications to the bearing. Examples are inductive or capacitive displacement sensors for slip and thermocouples and thermometers for temperature. ${ }^{4-11}$ Both in turn influence the actual rolling bearing operation due to the mass or contact introduced.

An alternative are non-contact measurement systems, such as high-speed cameras or thermal imaging cameras. With cameras, an unfavorable combination of rotational speed of the rotating bearing and exposure time of the camera results in motion blur, which complicates an analysis of the components. For high-speed cameras the shutter time and thus blur can be reduced by additional illumination. However, in thermal imaging cameras, the shutter time, also called integration time, depends on the temperature to be measured and cannot be reduced at will. Especially at high rotational speeds this leads to a limited applicability.

In the context of this paper, a derotator will therefore be used to extend contactless measuring devices intended for stationary objects to rotating ones. ${ }^{12}$ With this derotator it is possible to optically eliminate rotational movements. As a result, the image displayed by the derotator appears stationary despite the existing rotation. This phenomenon can then be exploited by various measuring devices, e.g. high-speed or thermal imaging cameras, to prevent motion blur. ${ }^{12-15}$ Here the derotator will be combined with the thermal imaging camera.

The functionality of the derotator is explained in the next section. This is followed in the third section by a description of the measurement procedures for the detection of cage slip and the temperature in the bearing. In the fourth section, the measurement results generated with the aid of these sequences are presented. The last section provides a summary and an outlook.

\section{FUNCTIONALITY OF THE DEROTATOR}
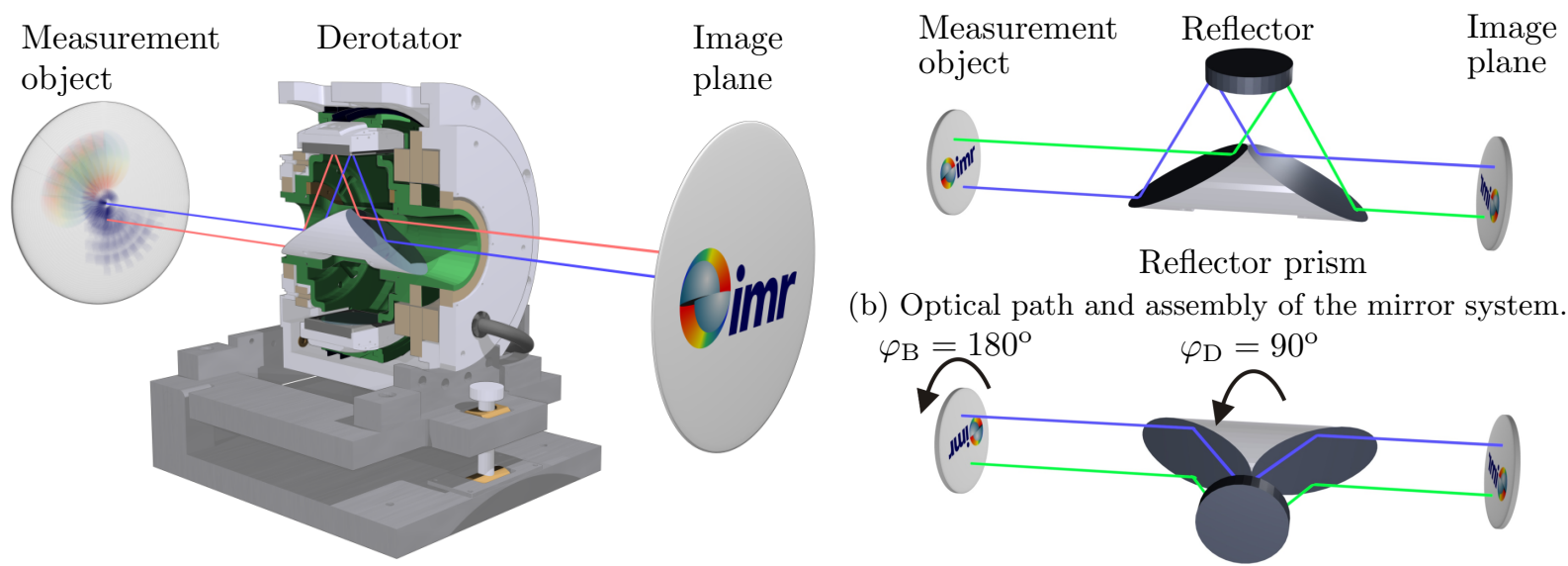

(b) Optical path and assembly of the mirror system.

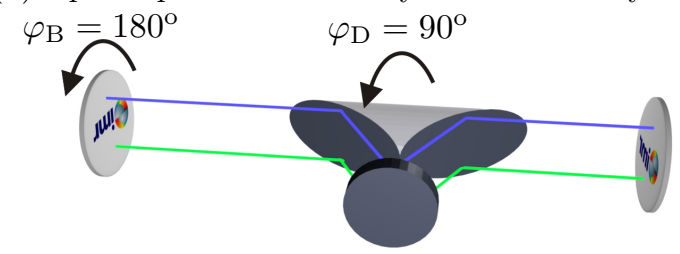

(a) Optical path from the measurement object to the image plane (c) Effect of a rotation $\varphi_{\mathrm{B}}=180^{\circ}$ and $\varphi_{\mathrm{D}}=90^{\circ}$ on via the reflector system inside the derotator. the optical path and image.

Figure 1: Optical principle of the derotator.

The optical elimination of the rotational movement of rotating measurement objects, e.g. a bearing, by means of the derotator is made possible by the use of a rotator set in rotation. A hollow-shaft synchronous motor is used to generate the rotary motion, whose rotation angle $\varphi_{\mathrm{D}}$ and angular velocity $\omega_{\mathrm{D}}$ can be read out by means of a rotary encoder. In the center of the derotator and the hollow shaft motor is the rotator, which reflects light 
rays coming from the measurement object, so that the image can be captured by the different measuring devices in the image plane (cf. Fig. 1a).

For the derotator used in the context of this work, the rotator is made of an aluminum alloy reflector system. ${ }^{12}$ It consists of a plane mirror and a double oblique plane mirror, so that light rays experience a total of three reflections. Otherwise it is common to manufacture the rotator as a glass prism. ${ }^{16}$ The reflector system used in this work enables both visible light and infrared radiation to be reflected and enables high mechanical strength. ${ }^{12}$

Two conditions must be fulfilled for the derotator to generate standing images: First, the axis of rotation of the derotator and that of the inner ring of the rolling bearing must be coaxial. Second, the rotation angle and the angular velocity of the derotator must be half the angle/angular velocity of the rolling bearing, meaning $\varphi_{\mathrm{D}}=0.5 \cdot \varphi_{\mathrm{B}}$ and $\omega_{\mathrm{D}}=0.5 \cdot \omega_{\mathrm{B}}$ (cf. Fig. $1 \mathrm{~b}$ and $1 \mathrm{c}$ ). For this purpose, solutions were developed in earlier papers, which are used in this work. ${ }^{17-19}$ Therefore the derotator can be used in combination with optical, contactless measuring devices to analyze the bearing with respect to cage slip and temperature behavior.

\section{MEASURING METHODS FOR ROLLING BEARING BEHAVIOR}

A cylindrical roller bearing of type NU208 (cf. Fig. 2) is used as a demonstrator for the following measuring methods and experiments. The test bearing is fixed in a bearing test chamber by means of a sleeve against a shaft shoulder. The bearing can be loaded with different loads $P$ by means of a force application mechanism. The load can be quantified by a piezoelectric force sensor. The bearing is fitted so that the inner ring is pressed onto the shaft and is driven by a synchronous motor equipped with a rotary encoder, which allows the angular velocity of the inner ring $\omega_{\mathrm{i}}$ to be varied selectively and read out. The outer ring is stationary. In the following, the aim is to apply a method for determining cage slip using the high-speed camera and a method for determining temperature rise using the thermal imaging camera and the derotator.

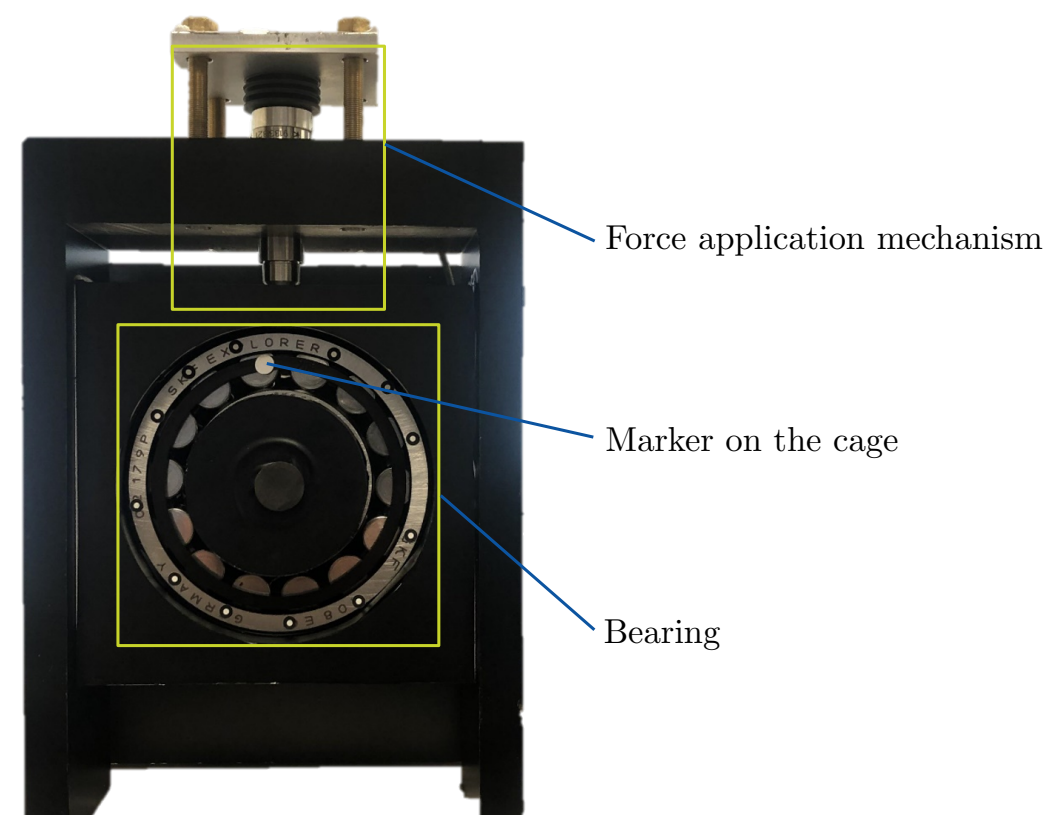

Figure 2: Rolling bearing test stand with the bearing NU208, the force application mechanism and the marker on the cage for the angular velocity determination.

Cage slip is calculated as

$$
S_{\mathrm{c}}=\frac{\omega_{\mathrm{c}}-\tilde{\omega}_{\mathrm{c}}}{\omega_{\mathrm{c}}}
$$


from the measured angular velocity $\tilde{\omega}_{\mathrm{c}}$ and the kinematically ideal angular velocity $\omega_{\mathrm{c}}$. The kinematically ideal angular velocity is calculated as

$$
\omega_{\mathrm{c}}=\frac{1}{2} \cdot \omega_{\mathrm{i}} \cdot \frac{d_{\mathrm{i}}}{d_{\mathrm{i}}+d_{\mathrm{r}}}
$$

from the inner ring diameter $d_{\mathrm{i}}$ and the rolling element diameter $d_{\mathrm{r}}$. The diameters specified by the manufacturer are used, but they may vary slightly due to the deflection during installation. The inner ring's angular velocity $\omega_{\mathrm{i}}$ is known from the encoder. Therefore, the cage's angular velocity $\tilde{\omega}_{\mathrm{c}}$ must be further determined.

To this end the high-speed camera is used. Since the cage is uniform in appearance and contains hardly any natural distinctive features, a white marker is applied to the cage (cf. Fig. 2). Compared to the weight of the bearing, the influence of the marker is negligible. Using segmentation algorithms, the marker is reduced to its center point $\left(x_{\mathrm{m}}, y_{\mathrm{m}}\right)^{\mathrm{T}}$. The marker can then be detected in all subsequent images. If the center of the cage $\left(x_{\mathrm{c}}, y_{\mathrm{c}}\right)^{\mathrm{T}}$ is set to $(0,0)^{\mathrm{T}}$ the angle between two images $k$ and $(k-y)$ can be calculated as

$$
\Delta \varphi_{\mathrm{c}}=\tan ^{-1}\left(\frac{y_{\mathrm{m}}(k-1)}{x_{\mathrm{m}}(k-1)}\right)-\tan ^{-1}\left(\frac{y_{\mathrm{m}}(k)}{x_{\mathrm{m}}(k)}\right) .
$$

With a given sampling time $T_{\mathrm{s}}$ of the image processing algorithm between two images, the angular velocity yields from Eq. 3 as

$$
\tilde{\omega}_{\mathrm{c}}=\Delta \varphi_{\mathrm{c}} \cdot T_{\mathrm{s}}^{-1} \text {. }
$$

By inserting Eqs. 2 and 4 into Eq. 1, the cage slip of the rolling bearing is obtained.

Next, the temperature changes of different bearing components during operation are determined. The heat flow generated through friction in a grease lubricated cylindrical roller bearing is given by

$$
\dot{Q}_{\mathrm{f}}=1.047 \cdot 10^{-4} \cdot n_{\mathrm{i}} \cdot M_{\mathrm{f}}
$$

and depends on its rotational speed $n_{\mathrm{i}}$ and the frictional torque $M_{\mathrm{f}}$. Hereby $M_{\mathrm{f}}$ is a function of the bearing's geometric dimensions as well as its rotational speed $n_{\mathrm{i}}$, its load $P$ and the lubricant's kinematic viscosity $\nu .{ }^{20}$

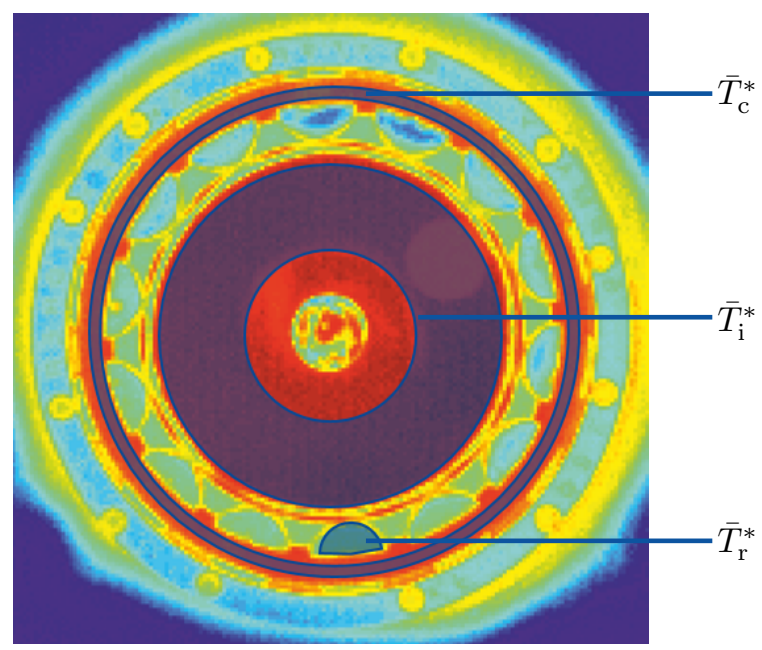

Figure 3: Regions of interest (ROIs) of which the averaged temperature increase $\bar{T}^{*}$ based on $\epsilon=1$ is calculated $\left(\bar{T}_{\mathrm{c}}^{*}\right.$ : cage, $\bar{T}_{\mathrm{i}}^{*}$ : inner ring, $\bar{T}_{\mathrm{r}}^{*}$ : rolling element).

To measure the temperature change the thermal imaging camera is used. As described above, this is difficult to do without a derotator, as the integration time of the camera without a derotator causes motion blur. With a thermal imaging camera the emitted heat of a measuring object is measured. The emitted radiation

$$
\dot{Q}_{\mathrm{e}}=\epsilon \cdot \sigma \cdot A \cdot\left(T_{\mathrm{b}}-T_{\mathrm{a}}\right)^{4}
$$


from surface $A$ with emissivity $\epsilon$ into a much larger surrounding space depends on the difference between the temperature $T_{\mathrm{b}}$ of the bearing and the ambient temperature $T_{\mathrm{a}}$ to the power of $4, \sigma$ is the Stefan-Boltzmann constant. The emissivity is a value between 0 and 1 , where 0 is the emissivity for an ideal mirror and 1 is the value for an ideal black body. Since the emissivity of the rolling bearing components is not known, only temperature differences are considered in the following, whereby these are based on the assumption that the emissivity is $\epsilon=1$.

The measurements are therefore not absolute temperature differences since the individual components have different surface finishes (cf. Fig. 2). For instance, the rolling elements have a silver mirror finish and the cage and sleeve on the inner ring are black, so a comparison between the components is not yet possible. Since the surface of the component itself does not change, it is still possible to draw conclusions about relative changes in the heat radiation emitted under different operating conditions.

The temperature increase $\Delta T^{*}$ based on $\epsilon=1$ is first determined by defining a region of interest (ROI) for each component (rolling element, cage, sleeve on the inner ring: cf. Fig. 3), of which the initial mean value $\bar{T}_{\mathrm{ROI}, 0}^{*}$ is calculated. This can be subtracted from the measured average temperature $\bar{T}_{\mathrm{ROI}}^{*}$ in the following measurements to determine the temperature increase

$$
\Delta \bar{T}_{\mathrm{ROI}}^{*}=\bar{T}_{\mathrm{ROI}}^{*}-\bar{T}_{\mathrm{ROI}, 0}^{*}
$$

This averaging makes it possible to determine the averaged increase in temperature of the inner ring and cage without a derotator in the case of motion blur. However, if the rolling elements or, in the future, local temperature peaks are investigated, the use of the derotator is essential for a spatially resolved indication of the temperature.

\section{EXPERIMENTAL RESULTS}

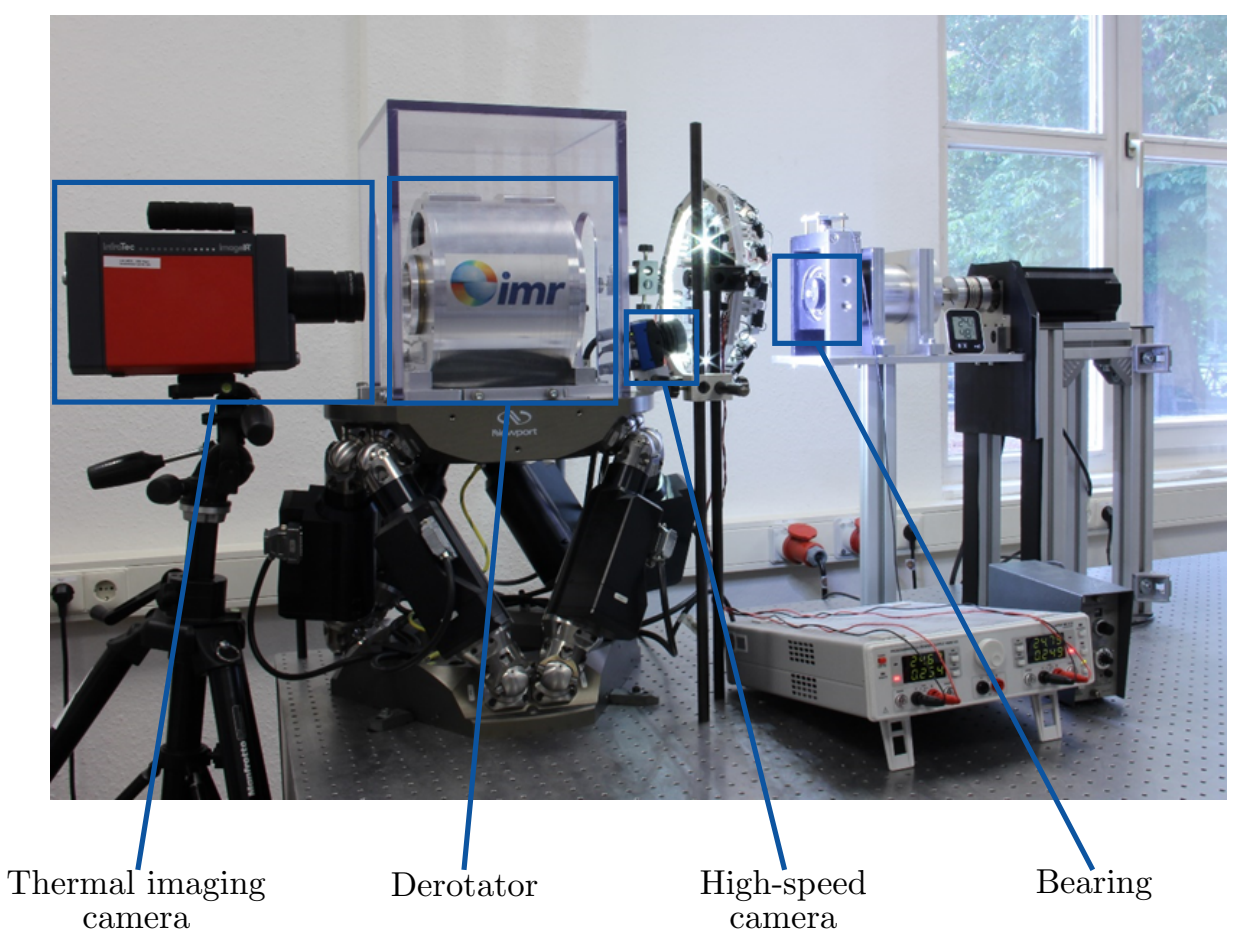

Figure 4: Test rig with the derotator, cylindrical roller bearing and both measurement instruments (thermal imaging camera and high-speed camera).

In this section the experimental results using both procedures described above will be discussed. To this end the test rig as shown in Fig. 4 is used. The camera is operated with a $18 \mathrm{~mm}$ lens at $400 \mathrm{fps}$ with $800 \mu$ s exposure 
time with the highest possible aperture $(f / 4)$ in order to prevent motion blur and to obtain an image as bright as possible. The thermal imaging camera is operated with a $25 \mathrm{~mm}$ lens at $1 \mathrm{fps}$ with $3200 \mu$ s exposure time.

As the load $P$ and the rotational speed $n_{\mathrm{i}}$ have an influence on the generated heat flow through friction (cf. Eq. 5)) as well as on slip, both are varied in the experiments. The load $P$ is varied between $0 \mathrm{~N}, 150 \mathrm{~N}$ and $350 \mathrm{~N}$ (minimum load). The rotational speed of the inner ring is varied between $148 \mathrm{rpm}, 444 \mathrm{rpm}$, and $740 \mathrm{rpm}$ (which corresponds to cage's rotational speeds of $60 \mathrm{rpm}, 180 \mathrm{rpm}$ and $300 \mathrm{rpm}$ if no slip is present). The bearing is operated for $600 \mathrm{~s}$ for each of the nine load/speed-combinations. A cooling period of 24 hours is allowed between each measurement. The results from the measurements are given in Tab. 1 and 2.

Table 2: Temperature difference $\Delta T_{600 \text { s }}^{*}$ after 600 $\mathrm{s}$ for the different loads and rotational speeds.

Table 1: Mean cage slip $\bar{S}_{\mathrm{c}}$, empirical standard deviation $s_{\bar{S}_{\mathrm{c}}}$ describing the scattering of the mean value and the slope $m_{\bar{S}_{\mathrm{c}}}$ of the regression line of the averaged cage slip curve.

\begin{tabular}{|c|c|c|c|c|}
\hline $\begin{array}{l}n_{\mathrm{i}} \\
\text { in rpm }\end{array}$ & $\begin{array}{l}F_{\mathrm{r}} \\
\text { in } \mathrm{N}\end{array}$ & $\begin{array}{l}\bar{S}_{\mathrm{c}} \\
\cdot 10^{-3}\end{array}$ & $\begin{array}{l}s_{\bar{S}_{\mathrm{c}}} \\
\cdot 10^{-3}\end{array}$ & $\begin{array}{l}m_{\bar{S}_{\mathrm{c}}} \\
\cdot 10^{-7}\end{array}$ \\
\hline \multirow{3}{*}{148} & 0 & 4.358 & 0.259 & -3.324 \\
\hline & 150 & 6.547 & 0.246 & 5.316 \\
\hline & 350 & 7.69 & 0.259 & -2.325 \\
\hline \multirow{3}{*}{444} & 0 & 4.982 & 0.160 & -1.255 \\
\hline & 150 & 8.211 & 0.196 & 6.951 \\
\hline & 350 & 8.779 & 0.164 & 1.287 \\
\hline \multirow{3}{*}{740} & 0 & 6.255 & 0.129 & -2.769 \\
\hline & 150 & 8.411 & 0.165 & 1.776 \\
\hline & 350 & 8.737 & 0.177 & -1.572 \\
\hline
\end{tabular}

\begin{tabular}{l|l|l|l}
$n_{\mathrm{i}}$ & $F_{\mathrm{r}}$ & Compo- & $\Delta T_{600 \mathrm{~s}}^{*}$ \\
in rpm & in N & nent & in ${ }^{\circ} \mathrm{C}$ \\
\hline \hline \multirow{4}{*}{148} & 0 & sleeve & 0.581 \\
& 0 & cage & 0.498 \\
& 0 & roller & 0.333 \\
& 150 & sleeve & 0.722 \\
& 150 & cage & 0.613 \\
& 150 & roller & 0.276 \\
& 350 & sleeve & 0.658 \\
& 350 & cage & 0.574 \\
& 350 & roller & 0.467 \\
\hline \multirow{4}{*}{444} & 0 & sleeve & 1.418 \\
& 0 & cage & 0.879 \\
& 0 & roller & 0.451 \\
& 150 & sleeve & 1.477 \\
& 150 & cage & 0.989 \\
& 150 & roller & 0.425 \\
& 350 & sleeve & 1.821 \\
& 350 & cage & 1.665 \\
& 350 & roller & 0.927 \\
\hline \multirow{6}{*}{740} & 0 & sleeve & 1.864 \\
& 0 & cage & 1.256 \\
& 0 & roller & 0.512 \\
& 150 & sleeve & 2.201 \\
& 150 & cage & 1.349 \\
& 150 & roller & 0.427 \\
& 350 & sleeve & 3.213 \\
& 350 & cage & 3.103 \\
& 350 & roller & 0.507
\end{tabular}

In the case of cage slip, it is noticeable that the overall cage slip $\bar{S}_{\mathrm{c}}$ is always negligibly small, because compared to Eq. 1 cage slip is a value between 0 (no slip) and 1 (maximum slip). Furthermore, it can be seen that the slip increases slightly with increasing rotational speed and load. Related to the rotational speed this confirms the results from the literature, related to the load it contradicts the literature. However, the slip can be influenced by other factors, e.g. the deviation of the installation diameters or a progressive lack of lubrication due to the use of the same test bearing for all experiments. The empirical standard deviation $s_{\bar{S}_{\mathrm{c}}}$ describing the scattering of the mean value as well as the slope of a regression line of the averaged cage slip indicate that the cage slip is constant over the $600 \mathrm{~s}$.

Generally speaking, investigations of the temperature difference show an increase in temperature rise with increasing rotational speed and load. With increasing rotational speed, a clear increase in temperature rise can be seen. With increasing load this is also visible, but with much less influence. Deviations from this trend 
can be seen in individual cases. Since no cage slip could be determined, this increase results either from the expected heating of the bearing according to Eq. 5 or from other harmful rolling bearing conditions. As already mentioned, a comparison of the components is not useful due to the lack of emissivity correction.

\section{SUMMARY AND OUTLOOK}

In summary, it can be said that the derotator enables rotating components to be examined with different measuring instruments. In this case, this was used to measure the temperature increase of a rolling bearing during operation by means of a contactless thermal imaging camera. Furthermore, cage slip was measured simultaneously with the help of a high-speed camera. The goal was to investigate a potential correlation between an increase in heating and cage slip. For this reason, series of measurements were carried out under different load and rotational speed conditions. Under all conditions, however, only negligibly small slip occurred, so that such a correlation could not yet be measured. Overall, the temperature increase is largely as expected.

In the future, it is reasonable to operate the rolling bearing under further operating conditions in which cage slip occurs. Thus it can be analyzed whether a stronger increase of the bearing temperature occurs in these operating modes. Furthermore, with the derotator it is not only possible to determine an average temperature rise, but also to examine the temperature distribution of individual components. In addition with a precise determination of the emissivity, this can increase the knowledge gained about harmful slip. Since cage slip is not the only potentially damaging rolling bearing behavior that can lead to increased friction and temperature, it is desirable to measure and differentiate other behavior such as cage whirl or rolling element slip simultaneously.

\section{REFERENCES}

[1] Schaeffler Technologies AG \& Co. KG, ed., [Wälzlagerpraxis: Handbuch zur Gestaltung und Berechnung von Wälzlagerungen], Vereinigte Fachverl., Mainz (2015).

[2] Bhushan, B., [Modern tribology handbook], CRC Press, Boca Raton, FL (2001).

[3] Tassone, B. A., "Roller bearing slip and skidding damage," Journal of Aircraft 12(4), 281-287 (1975).

[4] van Lier, H., Hentschke, C., and Jacobs, G., "Schädlicher Wälzlagerschlupf: Wann ist Wälzlagerschlupf schädlich und führt zum Ausfall des Wälzlagers? ; Abschlussbericht."

[5] Kacmarsky, W. M. and Hewko, L. O., "Effect of a High Traction Fluid on Skidding in a High Speed Roller Bearing," Journal of Lubrication Technology 93(1), 11 (1971).

[6] Wiemer, M., Theoretische und experimentelle Untersuchungen zum Betriebsverhalten vollrolliger Zylinderrollenlager, Thesis (Ph.D.), Leibniz Universität, Hannover (1990).

[7] Marquart, M., Neubauer, T., and Gerken, J.-D., [Leistungsdichte Wälzlager: Quantifizierung von Leistungsdichtegrenzen von Wälzlagern zur Vermeidung von Drehzahlschäden], FVA Forschungsvereinigung Antriebstechnik e.V (2011).

[8] Evans, R. D., Barr, T. A., Houpert, L., and Boyd, S. V., "Prevention of Smearing Damage in Cylindrical Roller Bearings," Tribology Transactions 56(5), 703-716 (2013).

[9] Selvaraj, A. and Marappan, R., "Experimental analysis of factors influencing the cage slip in cylindrical roller bearing," The International Journal of Advanced Manufacturing Technology 53(5-8), 635-644 (2011).

[10] Takabi, J. and Khonsari, M. M., "Experimental testing and thermal analysis of ball bearings," Tribology International 60, 93-103 (2013).

[11] Brecher, C., Fey, M., Hassis, A., and Bonerz, S., eds., [High-Speed Rolling Bearing Test Rigs with Contactless Signal Transmission for Measuring the Inner Ring Temperature] (2014).

[12] Mirzaei, S., Abo-Namous, O., Beichert, G., Fahlbusch, T., and Reithmeier, E., "Developing a new generation of optomechanical derotator for analysis of the dynamic behaviour of rotating components," in [Advances in Optomechanics], Hatheway, A. E., ed., SPIE Proceedings, 74240I, SPIE (2009).

[13] Altmann, B., Pape, C., and Reithmeier, E., "Temperature measurements on fast-rotating objects using a thermographic camera with an optomechanical image derotator," in [Infrared Sensors, Devices, and Applications VII], LeVan, P. D., Sood, A. K., Wijewarnasuriya, P., and D’Souza, A. I., eds., 29, SPIE (2017). 
[14] Altmann, B., Pape, C., and Reithmeier, E., "Digital image processing algorithms for automated inspection of dynamic effects in roller bearings," in [Automated Visual Inspection and Machine Vision II], Beyerer, J. and Puente León, F., eds., 103340A, SPIE (2017).

[15] Reithmeier, E., Mirzaei, S., and Kasyanenko, N., "Optical vibration and deviation measurement of rotating machine parts," Optoelectronics Letters 4(1), 45-48 (2008).

[16] Swift, D. W., "Image rotation devices - a comparative survey," Optics 8 Laser Technology 4(4), 175-188 (1972).

[17] Rohloff, B. S., Pape, C., and Reithmeier, E., "Automated calibration of an optomechanical derotator using 6-axes parallel kinematics and industrial image processing algorithms," Optical Engineering 53(10), 104101 (2014).

[18] Altmann, B., Betker, T., Pape, C., and Reithmeier, E., "Alignment strategy for an optomechanical image derotator using a laser Doppler vibrometer," Applied optics 58(24), 6555-6568 (2019).

[19] Altmann, B., Pape, C., and Reithmeier, E., "Image-based discrete-time optimal feedback control for an optomechanical derotator," IFAC-PapersOnLine 51(32), 292-297 (2018).

[20] Palmgren, A., "Neue Untersuchungen uber Energieverluste in Walzlagern," Wälzlagertechnische Mitteilungen (44), 117-121 (1957). 